\title{
Assessment of left atrial function by strain in patients with acute ischemic stroke left atrial function and acute stroke
}

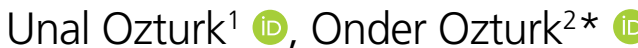

\section{SUMMARY}

OBJECTIVE: Myocardial speckle-tracking echocardiography can detect subtle abnormalities in the left atrial function. In this study, we aimed to investigate the relationship between left atrial myocardium and tissue function $n$ assessed by two-dimensional speckle-tracking echocardiography and the National Institutes of Health Stroke Scale score in patients with acute ischemic stroke.

METHOD: The study was composed of 80 patients ( 45 men, 35 women, mean age: $67 \pm 15$ years) with acute ischemic stroke. The patients were divided into two groups based on the calculated National Institutes of Health Stroke Scale score (group 1, National Institutes of Health Stroke Scale score < 16; group 2, National Institutes of Health Stroke Scale score $\geq 16$ ). Demographic, clinical, and laboratory data for all patients were collected. Cardiac functions were evaluated using two-dimensional speckle-tracking echocardiography within 48 hours from admission to the neurology care unit.

RESULTS: There were no significant differences between the patients' clinical parameters. Left ventricular ejection fraction was significantly higher in group 1 than in group $2(59.2 \pm 5.6$ to $51.4 \pm 6.3, p=0.024)$. Left atrial longitudinal strain was significantly higher in group 1 than in group $2(34.48 \pm 9.73$ to $26.27 \pm 7.41, p=0.019)$. There were no significant differences between other echocardiographic parameters. CONCLUSION: Our results suggest that left atrial longitudinal strain is associated with stroke severity during admission in patients with acute ischemic stroke. Left atrial longitudinal strain is an indicator of left atrial myocardial function.

KEYWORDS: Atrial function. Heart atria. Echocardiography. Stroke.

\section{INTRODUCTION}

Acute stroke is an important cause of morbidity and mortality $^{1,2}$. Cardiovascular complications are common after an acute stroke ${ }^{3}$. Increasing sympathetic activation leads to myocardial injury. Neurogenic stress cardiomyopathy (NSC) is a condition of acute left ventricular systolic dysfunction caused by acute neurological diseases, such as acute ischemic or hemorrhagic stroke, cranial trauma, intracranial hemorrhage, and epilepsy ${ }^{4}$. However, there is no significant obstruction of the coronary arteries in patients with NSC. Acute systolic heart failure caused by NSC is an important cause of lethal ventricular dysrhythmia and mortality ${ }^{4}$.
Echocardiography is most commonly used for the assessment of cardiovascular functions in acute ischemic stroke (AIS) patients. Few authors have demonstrated that left atrial enlargement (LAE) is independently associated with new neurological events in patients with first-ever AIS. Assessment of left atrial (LA) function is important in acute stroke patients ${ }^{5,6}$.

Two-dimensional (2D) speckle-tracking echocardiography (STE) allows for the assessment of global and local myocardial tissue function ${ }^{7}$. It calculates the regional ratio of myocardial tissue deformation. This novel echocardiographic technique evaluates the left ventricular (LV) myocardium and tissue.

1 University of Health Sciences, Diyarbakır Gazi Yasargil Education and Research Hospital, Department of Neurology - Diyarbakir, Turkey. 2University of Health Sciences, Diyarbakır Gazi Yasargil Education and Research Hospital, Department of Cardiology - Diyarbakir, Turkey. *Corresponding author: droozturk21@hotmail.com

Conflicts of interest: the authors declare there are no conflicts of interest. Funding: none. Received on September 6, 2020. Accepted on September 12, 2020. 
However, this technique has seldom evaluated the tissue function and the atrial myocardium ${ }^{8}$.

In our study, we aimed to investigate the association between the tissue function and the atrial myocardium assessed by 2D-STE and National Institutes of Health Stroke Scale (NIHSS) score in AIS patients.

\section{METHODS}

\section{Study participants and design}

\section{Patient selection}

We prospectively studied 97 consecutive patients (males, 42; females, 55; mean age, $65 \pm 16$ years; range $41-80$ years) within $\leq 24$ hours from the onset of AIS symptoms admitted to the neurology care unit, between October 2016 and December 2017. Seventeen patients were excluded. The patients' demographic and baseline clinical characteristics, including the severity assessment of neurological deficit with NIHSS upon admission to the neurology care unit, were recorded. Patients with a well-defined time of symptom onset of acute ischemic stroke were included in this study. Baseline stroke severity was evaluated using the NIHSS score?

All patients underwent immediate computed tomography after being admitted to the emergency care unit. Troponin values were calculated, and an electrocardiogram (ECG) was recorded after admission to the neurology intensive care unit. Echocardiography was performed within the first 48 hours after admission. The study was certified by our hospital's Ethics Committee. Informed consent was obtained from all patients.

\section{Echocardiographic examination}

All patients underwent detailed 2D transthoracic echocardiographic study at rest according to the echocardiographic recommendation ${ }^{9}$, using a Philips EPIQ 7C ultrasound system (Philips Healthcare, Andover, MA, USA) coupled with a multifrequency transducer $(3-8 \mathrm{MHz})$ and tissue harmonic imaging ability. A single-lead ECG was placed permanently during the study, and $2 \mathrm{D}$ echocardiographic images of the LA and LV were acquired in the apical four-chamber and two-chamber view at end-inspiration. The frame rate was adjusted between 60 and 80 frames per second. At least three successive cardiac cycles of $2 \mathrm{D}$ echocardiographic images in the apical four-chamber and two-chamber view were stored in order to choose the images with the best quality for off-line speckle-tracking analysis ${ }^{8}$.

\section{Two-dimensional speckle-tracking analysis}

Strain analysis was carried out by a skilled investigator who was blinded to the clinical parameters, using the latest commercially available $2 \mathrm{D}$ wall motion tracking software (Philips Healthcare Systems). For speckle-tracking analysis, apical fourand two-chamber view images were obtained using conventional 2D grayscale echocardiography, at end-inspiration with a stable ECG logger. Particular attention was paid to obtain an adequate grayscale image, allowing a reliable delineation of the myocardial tissue and the extracardiac structures. The average recording of three consecutive heart cycles was taken. The frame rate was set between 60 and 80 frames per second. These settings are recommended to combine temporal resolution with adequate spatial definition and to enhance the feasibility of the frame-to-frame tracking technique ${ }^{7}$.

To evaluate LA myocardial tissue function, LA endocardium was manually traced when the left atrium was at its maximum volume, just before mitral valve opening, as recognized in the apical four-chamber and two-chamber views. The LA basal septal, basal lateral and apical myocardial borders were manually traced, followed by automatic tracing of the LA endocardial and epicardial borders. This described an area of interest composed of six segments. Longitudinal strain curves were generated by the software for each atrial segment after analysis of segmental tracking quality and manual adjustment of the area of interest. LA longitudinal speckle tracking was calculated in six segments of the atrium (Figure 1$)^{8}$. Pathan et al. ${ }^{10}$ found a normal reference range of $39 \%$ for left atrial longitudinal strain (LALS) (38-41\%).

\section{Definition of Stroke and Assessment of Stroke Severity}

The updated definition of stroke in the American Heart Association/American Stroke Association guidelines states that ischemic stroke is diagnosed based on the combination of symptoms and/or signs of typical neurological dysfunction and imaging that is proof of cerebral infarction ${ }^{11}$.

The NIHSS is a simple, valid, and reliable systematic assessment tool that measures acute stroke-related neurological defi$\mathrm{cit}^{12}$. The higher the NIHSS score, the higher the impairment of a stroke patient. There are five stroke severity groups: NIHSS $=0$ (no stroke), NIHSS $=1-4$ (minor stroke), NIHSS $=5-15$ (moderate stroke), NIHSS=16-20 (moderate to severe stroke), and NIHSS $=21-42$ (severe stroke). A baseline NIHSS score $>16$ indicates a strong probability of patient disability and death ${ }^{12}$. Patients were categorized into two groups according to severity: group 1, composed of patients with non-severe stroke (NIHSS $<16 ; n=58$ ); group 2, composed of patients with severe stroke (NIHSS $\geq 16 ; n=22)$. 


\section{Cerebral Infarction Volume Measurements}

A neurolog computed the "cerebral infarction volume" in each patient by using Analyze 12.0, a software package for biomedical imaging analysis (Biomedical Imaging Resource, New York, NY, USA). The regions of interest were segmented

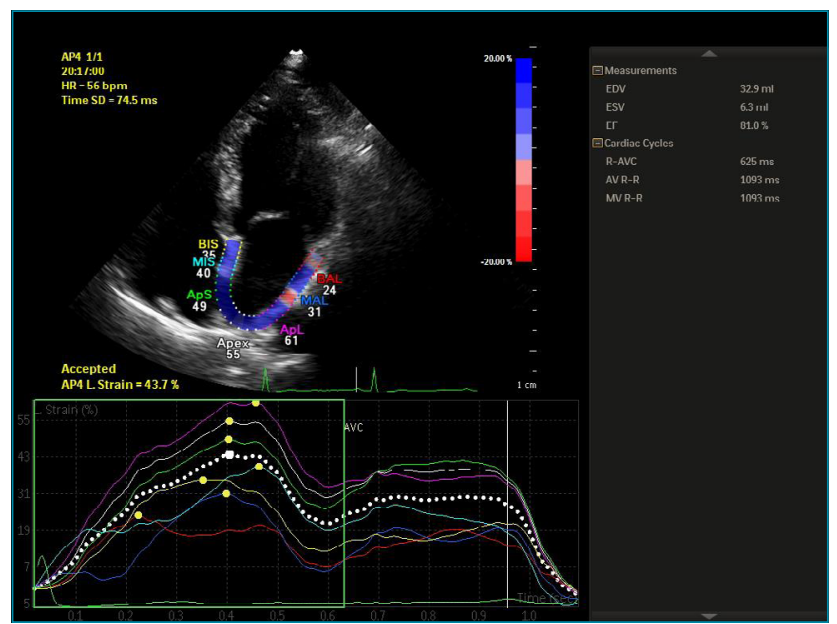

AVC: aortic valve closure.

Figure 1. Example of echo-derived left atrial strain using speckle tracking. Regional strains are denoted by the colored lines, longitudinal strain by the white dotted line. using the Region Grow in the Volume Edit module, with manual removal of artifacts when necessary. The total volume was reported in milliliters.

\section{Statistical analysis}

Statistical analysis was conducted with the SPSS statistical package (version 12.0; SPSS Inc., Chicago, IL, USA). All baseline parameters were analyzed. Continuous variables were expressed as mean $\pm \mathrm{SD}$; and categorical variables were expressed as percentage rates. Intraobserver variability was calculated as the absolute difference between the two measurements as a percentage of their mean. The Mann-Whitney U test and the Chi-square test were used appropriately for comparing data. P-values of $<0.05$ were considered as statistically significant. Pearson's correlation was used to determine the association between LA 2D-STE and other echocardiographic parameters. The Pearson's or Spearman's correlation was used for assessing correlations between variables.

\section{RESULTS}

\section{Baseline characteristics}

The patients' baseline characteristics are summarized in Table 1. Clinical characteristics of groups were similar with

Table 1. Patients' clinical characteristics.

\begin{tabular}{l|c|c|c} 
& $\begin{array}{c}\text { Group 1 } \\
\text { AglHSS (years) }\end{array}$ & $\begin{array}{c}\text { Group 2 } \\
\text { (NIHSS score } \geq 16) \mathrm{n}=22\end{array}$ & p-value \\
\hline Gender (F/M), $\mathrm{n}$ & $65.4 \pm 12.7$ & $69.8 \pm 17.7$ & 0.619 \\
\hline Hypertension, $(\mathrm{n} \%)$ & $32 / 26$ & $13 / 9$ & 0.718 \\
\hline SBP $(\mathrm{mmHg})$ & $28(48)$ & $13(59)$ & 0.062 \\
\hline DBP $(\mathrm{mmHg})$ & $143.5 \pm 15.2$ & $159.8 \pm 18.5$ & 0.043 \\
\hline Heart Rate $(\mathrm{bpm})$ & $76.4 \pm 8.6$ & $84.3 \pm 12.7$ & 0.048 \\
\hline Diabetes Mellitus, $(\mathrm{n} \%)$ & $92.3 \pm 14.7$ & $115 \pm 16.5$ & 0.027 \\
\hline Smoking, $(\mathrm{n} \%)$ & $14(24)$ & $6(29)$ & 0.091 \\
\hline Dyslipidemia, $(\mathrm{n} \%)$ & $7(12)$ & $4(16)$ & 0.076 \\
\hline Infarct volume $(\mathrm{mL})$ & $9(15)$ & $8(36)$ & 0.043 \\
\hline Troponin $(\mathrm{ng} / \mathrm{L})$ & $16 \mathrm{~mL} \pm 2.3$ & $44 \mathrm{~mL} \pm 4.1$ & 0.034 \\
\hline HbA1c $(\%)$ & 6.143 & 14.705 & 0.037 \\
\hline Glucose $(\mathrm{mg} / \mathrm{dL})$ & $6.24 \pm 1.43$ & $9.56 \pm 1.53$ & 0.009 \\
\hline Creatinine $(\mathrm{mg} / \mathrm{dL})$ & $143.5 \pm 37.4$ & $192.7 \pm 48.5$ & 0.026 \\
\hline LDL cholesterol $(\mathrm{mg} / \mathrm{dL})$ & $1.3 \pm 0.4$ & $1.8 \pm 0.6$ & 0.023 \\
\hline HDL cholesterol $(\mathrm{mg} / \mathrm{dL})$ & $103.3 \pm 27.1$ & $128.4 \pm 39.5$ & 0.008 \\
\hline
\end{tabular}

NIHSS: National Institutes of Health Stroke Scale. F: Female. M: Male. SBP: Systolic Blood Pressure. DBP: Diastolic Blood Pressure. LDL: Low Density Lipoprotein. HDL: High Density Lipoprotein. 
respect to age, gender, hypertension, diabetes, and smoking ( $\mathrm{p}$ $>0.05)$. Systolic blood pressure (BP), diastolic BP, heart rate, dyslipidemia, infarction volume, glucose, creatinine, and troponin levels in group 2 were significantly higher than group 1 $(\mathrm{p}<0.05)$. Serum hemoglobin HbA1c and low-density lipoprotein (LDL) cholesterol levels were significantly higher in group 2 than group $1(\mathrm{p}<0.05)$.

\section{Echocardiographic findings}

Echocardiographic findings are summarized in Table 2. LV wall thickness and E/e' values were significantly higher in group 2 than in group $1(\mathrm{p}<0.05)$. The absolute value of LALS and LVEF were significantly higher in group 1, with lower NIHSS scores, than in group 2, with higher NIHSS scores. Around 10 patients $(12 \%)$ were randomly assigned to assess intraobserver variability and it was calculated as $3.8 \%$.

Correlation analysis was performed to investigate the relationship between the NIHSS score and echocardiographic parameters. A negative correlation was found between the NIHSS score and LVEF and the absolute value of the LALS. There was a positive correlation between the absolute value of the NIHSS score and age, heart rate, and E/e' (Table 3). Logistic regression analysis was performed to identify the potential predictors for stroke severity. Results of the multivariate analysis revealed age, LVEF, and LALS as powerful predictors of severe ischemic stroke (Table 4).

\section{DISCUSSION}

Ischemic stroke and heart failure are important causes of morbidity and mortality. Heart failure is associated with an increased risk of ischemic stroke ${ }^{13}$. LV dysfunction occurs frequently after cerebrovascular events ${ }^{14}$. LVEF is a beneficial marker of systolic function; however, it was not detected in early LV systolic dysfunction. Global longitudinal strain (GLS) is an indicator of myocardial deformation, which is different from LVEF. GLS can detect early myocardial dysfunction despite the preserved $\mathrm{LVEF}^{15}$.

LV longitudinal strain is an important parameter of LA myocardial function. LV function has an effect on the systolic

Table 3. Correlation between the National Institutes of Health Stroke Scale score and clinical parameters in acute ischemic stroke patients.

\begin{tabular}{l|c|c} 
Parameters & $\begin{array}{c}\text { Pearson's correlation } \\
\text { coefficient (r value) }\end{array}$ & p-value \\
\hline LALS & -0.573 & 0.026 \\
\hline LVEF & -0.314 & 0.038 \\
\hline E/e' & 0.217 & 0.026 \\
\hline Age & 0.320 & 0.042 \\
\hline Heart rate & 0.419 & 0.023 \\
\hline
\end{tabular}

LALS: Left atrial longitudinal strain. LVEF: Left ventricular ejection fraction.

Table 2. Patients' echocardiographic parameters.

\begin{tabular}{|c|c|c|c|}
\hline & $\begin{array}{c}\text { Group } 1 \\
(\text { NIHSS score }<16) n=58\end{array}$ & $\begin{array}{c}\text { Group } 2 \\
\text { (NIHSS score } \geq 16) n=22\end{array}$ & p-value \\
\hline LV septal thickness, mm & $11.2 \pm 1.8$ & $12.7 \pm 1.7$ & 0.027 \\
\hline LVDd (mm) & $51.2 \pm 6.3$ & $54.6 \pm 6.8$ & 0.348 \\
\hline LV posterior Wall thickness, mm & $10.8 \pm 1.5$ & $11.9 \pm 1.6$ & 0.034 \\
\hline LVSs (mm) & $40.5 \pm 4.2$ & $43.2 \pm 5.8$ & 0.241 \\
\hline LVEDV (mL) & $86.0 \pm 17.7$ & $95.4 \pm 24.3$ & 0.192 \\
\hline LVESV (mL) & $41.1 \pm 12.4$ & $44.7 \pm 14.2$ & 0.246 \\
\hline $\mathrm{LAD}(\mathrm{mm})$ & $39.5 \pm 4.3$ & $42.4 \pm 4.6$ & 0.624 \\
\hline $\mathrm{RAD}(\mathrm{mm})$ & $32.6 \pm 3.4$ & $34.7 \pm 3.7$ & 0.590 \\
\hline RVDd (mm) & $28.1 \pm 2.5$ & $30.7 \pm 2.8$ & 0.369 \\
\hline LVEF (\%) & $59.2 \pm 5.6$ & $51.4 \pm 6.3$ & 0.024 \\
\hline LALS (\%) & $34.48 \pm 9.73$ & $26.27 \pm 7.41$ & 0.019 \\
\hline LV GLS (\%) & $-1.4 \pm 2.2$ & $-15.9 \pm 2.7$ & 0.003 \\
\hline$E / e^{\prime}$ & $8.7 \pm 2.9$ & $10.4 \pm 3.6$ & 0.017 \\
\hline
\end{tabular}

NIHSS: National Institutes of Health Stroke Scale. LV: Left Ventricle. LVDd: Left ventricular diastolic diameter. LVSs: Left ventricular systolic diameter. LVEDV: Left ventricular end-diastolic volume. LVESV: Left ventricular end-systolic volume. LAD: Left atrial diameter. RAD: Right atrial diameter. RVDd: Right ventricular diastolic diameter. LVEF: Left ventricular ejection fraction. LALS: Left atrial longitudinal strain. LV GLS: Left ventricular global longitudinal strain. 
Table 4. Multivariate logistic regression analysis between the National Institutes of Health Stroke scale score and clinical parameters in acute ischemic stroke patients.

\begin{tabular}{l|c|c|c} 
Parameters & OR & $95 \% \mathrm{Cl}$ & $\mathrm{p}$-value \\
\hline LALS & 0.571 & $0.289-0.752$ & 0.042 \\
\hline LVEF & 0.835 & $0.770-0.920$ & 0.025 \\
\hline Age & 1.218 & $1.090-1.465$ & 0.030 \\
\hline Heart rate & 1.130 & $0.972-1.223$ & 0.453 \\
\hline
\end{tabular}

OR: Odds ratio. LALS: Left atrial longitudinal strain. LVEF: Left ventricular ejection fraction.

descent of the mitral plane ${ }^{16}$. As a result, an impaired LV longitudinal strain causes a low LA longitudinal strain. The evaluation of LV longitudinal strain is an indirect calculation of LA myocardial function and compliance ${ }^{17}$.

Both atrial fibrillation (AF) and cardiovascular risk factors have been related with abnormalities of LA myocardial mechanical function ${ }^{18}$. The occurrence of strain imaging allows a sensitive evaluation of myocardial deformation, involving the analysis of LA myocardial function ${ }^{19}$. Impaired LA myocardial speckle tracking has been defined in patients with arterial hypertension and/or diabetes mellitus, but with normal LA diameter ${ }^{20}$. The Atherosclerosis Risk in Communities (ARIC) study demonstrated that LA size is an important predictor of cardiovascular death, morbidity, and mortality ${ }^{21}$.

LA myocardial STE is a beneficial marker for assessment of LA myocardial function. Very little is known about the association between AIS severity and LA myocardial function. Our study is the first-ever to assess LA function using 2D-STE in the early period of AIS. The novel and most important finding is that even mildly impaired LA function was detected with 2D-STE after AIS. We found that the absolute value of LALS was significantly higher in patients with lower NIHSS scores.

Lower LALS is reportedly associated with hypertension, LV hypertrophy, and diabetes mellitus ${ }^{22}$. We found that BP at admission is significantly higher in severe ischemic stroke patients. However, Bonardo et al. ${ }^{23}$ found that young patients with AIS and large infarction volume did not show high blood pressure at admission. We found that $\mathrm{LDL}$ cholesterol and $\mathrm{HbAlc}$ were significantly increased in patients with higher NIHSS scores. Hendrix et $\mathrm{a}^{24}$. found that history of diabetes mellitus was an important predictor of stroke severity.

Regardless of the previous cardiovascular situation, an early period of stroke prominently affects systemic BP, heart rate, LV function, and biochemical parameters (glucose, troponin, and creatinine $)^{14}$. Our study showed that troponin values were significantly higher in severe ischemic stroke patients. Chang et al..$^{25}$ observed that cardiac biomarkers, especially serum troponin levels, were related with acute large-vessel occlusion in ischemic stroke patients. Lindsberg et al. observed elevated blood glucose was frequent in the early period of the stroke ${ }^{26}$. Blood glucose levels were significantly higher in severe stroke patients during admission in our study. Also, E/é' value was significantly higher in severe stroke patients. Ryu et al. ${ }^{27}$ suggested that E/e' ratios were related with acute arterial occlusion in $\mathrm{AF}$-associated stroke and may play an important role in recognizing patients at high risk of severe stroke. Our study showed that creatinine levels were significantly higher in severe stroke patients. Mostofsky et al..$^{28}$ demonstrated that underlying risk factors for cardiovascular diseases, including age, diabetes mellitus, hypertension, and left ventricular hypertrophy, may represent an important vascular pathogenesis resulting from impaired renal clearance. Renal function is a very important predictor of survival in AIS patients.

\section{CONCLUSIONS}

The results of our study suggest that LALS is associated with stroke severity in AIS patients upon admission. LALS can evaluate LA myocardial function and stress in patients with acute neurological diseases. LALS can detect early atrial myocardial dysfunction. Therefore, this study suggests that LALS can detect early LA myocardial dysfunction in AIS patients.

\section{AUTHORS" CONTRIBUTIONS}

UO: Conceptualization, Data Curation, Formal Analysis, Writing - Original Draft, Writing - Review \& Editing. OO: Conceptualization, Data Curation, Formal Analysis, Writing - Original Draft, Writing - Review \& Editing.

\section{REFERENCES}

1. Ozturk U, Ozturk PA, Asena M. Magnesium as a risk factor and impact on survey in acute stroke. Dicle Med J. 2019;46(4):781-7. https://doi.org/10.5798/dicletip.661373

2. Rojek A, Gąsecki D, Fijałkowski M, Kowalczyk K, Kwarciany $M$, Wolf J, et al. Left ventricular ejection fraction and aortic stiffness are independent predictors of neurological outcome in acute ischemic stroke. J Hypertens. 2016;34(12):2441-8. https://doi.org/10.1097/HJH.0000000000001095

3. Malik AN, Gross BA, Rosalind Lai PM, Moses ZB, Du R. Neurogenic stress cardiomyopathy after aneurysmal 
subarachnoid hemorrhage. World Neurosurg. 2015;83(6):880-5. https://doi.org/10.1016/j.wneu.2015.01.013

4. Kerro A, Woods T, Chang JJ. Neurogenic stunned myocardium in subarachnoid hemorrhage. J Crit Care. 2017;38:27-34. https://doi.org/10.1016/j.jcrc.2016.10.010

5. Overvad TF, Nielsen PB, Larsen TB, Søgaard P. Left atrial size and risk of stroke in patients in sinus rhythm. A systematic review. Thromb Haemost. 2016;116(2):206-19. https://doi. org/10.1160/TH15-12-0923

6. Yaghi S, Moon YP, Mora-McLaughlin C, Willey JZ, Cheung K, Di Tullio MR, et al. Left atrial enlargement and stroke recurrence: the Northern Manhattan Stroke Study. Stroke. 2015;46(6):148893. https://doi.org/10.1161/STROKEAHA.115.008711

7. Amzulescu MS, De Craene M, Langet $H$, Pasquet $A$, Vancraeynest $D$, Pouleur AC, et al. Myocardial strain imaging: review of general principles, validation, and sources of discrepancies. Eur Heart J Cardiovasc Imaging. 2019;20(6):605-19. https:// doi.org/10.1093/ehjci/jez041

8. Gan GCH, Ferkh A, Boyd A, Thomas L. Left atrial function: evaluation by strain analysis. Cardiovasc Diagn Ther. 2018;8(1):29-46. https://doi.org/10.21037/cdt.2017.06.08

9. Lang RM, Badano LP, Mor-Avi V, Afilalo J, Armstrong A, Ernande $L$, et al. Recommendations for cardiac chamber quantification by echocardiography in adults: an update from the American Society of Echocardiography and the European Association of Cardiovascular Imaging. Eur Heart J Cardiovasc Imaging. 2015;16(3):233-71. https://doi.org/10.1093/ehjci/jev014

10. Pathan F, D'Elia N, Nolan MT, Marwick TH, Negishi K. Normal ranges of left atrial strain by speckle-tracking echocardiography: a systematic review and meta-analysis. J Am Soc Echocardiogr. 2017;30(1):59-70.e8. https://doi. org/10.1016/j.echo.2016.09.007

11. Sacco RL, Kasner SE, Broderick JP, Caplan LR, Connors JJ, Culebras A, et al. An updated definition of stroke for the 21st century: a statement for healthcare professionals from the american heart association/american stroke association. 2013;44(7):2064-89. https://doi.org/10.1161/STR.0b013e318296aeca

12. Lyden P. Using the National Institutes of Health Stroke Scale: a cautionary tale. Stroke. 2017;48(2):513-9. https://doi. org/10.1161/STROKEAHA.116.015434

13. Vemmos K, Ntaios G, Savvari P, Vemmou AM, Koroboki E, Manios E, et al. Stroke aetiology and predictors of outcome in patients with heart failure and acute stroke: a 10-year follow-up study. Eur J Heart Fail. 2012;14(2):211-8. https:// doi.org/10.1093/eurjhf/hfr172

14. Ripoll JG, Blackshear JL, Díaz-Gómez JL. Acute cardiac complications in critical brain disease. Neurosurg Clin N Am. 2018;29(2):281-97. https://doi.org/10.1016/j.nec.2017.11.007

15. Feigenbaum H, Mastouri R, Sawada S. A practical approach to using strain echocardiography to evaluate the left ventricle. Circ J. 2012;76(7):1550-5. https://doi.org/10.1253/circj. cj-12-0665

16. Barbier P, Solomon SB, Schiller NB, Glantz SA. Left atrial relaxation and left ventricular systolic function determine left atrial reservoir function. Circulation. 1999;100(4):427-36. https://doi.org/10.1161/01.cir.100.4.427
17. Skaarup KG, Christensen H, Høst N, Mahmoud MM, Ovesen C, Olsen FJ, et al. Usefulness of left ventricular speckle tracking echocardiography and novel measures of left atrial structure and function in diagnosing paroxysmal atrial fibrillation in ischemic stroke and transient ischemic attack patients. Int J Cardiovasc Imaging. 2017;33(12):1921-9. https://doi. org/10.1007/s10554-017-1204-1

18. Leong DP, Joyce E, Debonnaire P, Katsanos S, Holman ER, Schalij MJ, et al. Left atrial dysfunction in the pathogenesis of cryptogenic stroke: novel insights from speckle-tracking echocardiography. J Am Soc Echocardiogr. 2017;30(1):71-9. e1. https://doi.org/10.1016/j.echo.2016.09.013

19. Leong DP, Penhall A, Perry R, Shirazi M, Altman M, Chong D, et al. Speckle-tracking strain of the left atrium: a transoesophageal echocardiographic validation study. Eur Heart J Cardiovasc Imaging. 2013;14(9):898-905. https://doi.org/10.1093/ehjci/ jes323

20. Mondillo S, Cameli M, Caputo ML, Lisi M, Palmerini E, Padeletti $M$, et al. Early detection of left atrial strain abnormalities by speckle-tracking in hypertensive and diabetic patients with normal left atrial size. J Am Soc Echocardiogr. 2011;24(8):898908. https://doi.org/10.1016/j.echo.2011.04.014

21. Nagarajarao HS, Penman AD, Taylor HA, Mosley TH, Butler $\mathrm{K}$, Skelton TN, et al. The predictive value of left atrial size for incident ischemic stroke and all-cause mortality in African Americans: the Atherosclerosis Risk in Communities (ARIC) Study. Stroke. 2008;39(10):2701-6. https://doi.org/10.1161/ STROKEAHA.108.515221

22. Jarasunas J, Aidietis A, Aidietiene S. Left atrial strain - an early marker of left ventricular diastolic dysfunction in patients with hypertension and paroxysmal atrial fibrillation. Cardiovasc Ultrasound. 2018;16(1):29. https://doi.org/10.1186/s12947018-0147-6

23. Bonardo P, Pantiú F, Ferraro M, Chertcoff A, Bandeo L, Cejas $\mathrm{LL}$, et al. Impact of infarct size on blood pressure in young patients with acute stroke. J Vasc Interv Neurol. 2018;10(1):14-6. PMID: 29922398

24. Hendrix P, Sofoluke N, Adams MD, Kunaprayoon S, Zand R, Kolinovsky AN, et al. Risk factors for acute ischemic stroke caused by anterior large vessel occlusion. Stroke. 2019;50(5):1074-80. https://doi.org/10.1161/STROKEAHA.118.023917

25. Chang A, Ricci B, Grory BM, Cutting S, Burton T, Dakay K, et al. Cardiac biomarkers predict large vessel occlusion in patients with ischemic stroke. J Stroke Cerebrovasc Dis. 2019;28(6):1726-31. https://doi.org/ 10.1016/j.jstrokecerebrovasdis.2019.02.013

26. Lindsberg PJ, Roine RO. Hyperglycemia in acute stroke. Stroke. 2004;35(2):363-4. https://doi.org/10.1161/01. STR.0000115297.92132.84

27. Ryu WS, Bae EK, Park SH, Jeong SW, Schellingerhout D, Nahrendorf $M$, et al. Increased left ventricular filling pressure and arterial occlusion in stroke related to atrial fibrillation. J Stroke Cerebrovasc Dis. 2018;27(5):1275-82. https://doi. org/10.1016/j.jstrokecerebrovasdis.2017.12.009

28. Mostofsky E, Wellenius GA, Noheria A, Levitan EB, Burger MR, Schlaug $G$, et al. Renal function predicts survival in patients with acute ischemic stroke. Cerebrovasc Dis. 2009;28(1):8894. https://doi.org/10.1159/000219302 